\title{
Design and Evaluation of Identifiable Key-Click Signals for Mobile Devices
}

\author{
Hsiang-Yu Chen, Jaeyoung Park, Steve Dai, and Hong Z. Tan, Senior Member, IEEE
}

\begin{abstract}
As touch based input becomes more popular in mobile devices, there is an increasing need for haptic feedback on key-less input surface. Four experiments were conducted to design and evaluate identifiable emulated key-click signals using a piezoelectric actuator. Experiments I and II assessed the information transmission capacity for the amplitude, frequency, and number of cycles of raised cosine waveforms used to drive the piezo actuators under fixed- and roving-background conditions, respectively. Experiment III estimated the total information transfer for all three parameters. The results were used to reduce the number of stimulus alternatives in the key-click signal set with the goal to achieve perfect identification performance. Experiment IV verified that up to 5 to 6 identifiable key-click signals could be achieved with the experimental setup. The present study outlines an information theoretic approach to conducting identification experiments to guide the design of and to evaluate a perfectly identifiable stimulus set. The methodology can be applied to other applications in need of perceptually identifiable stimulation patterns.
\end{abstract}

Index Terms-Mobile applications, haptic feedback, key click, human information processing.

\section{INTRODUCTION}

$\mathrm{H}$ APTIC interactions have become increasingly popular in consumer products in the last decade. Applications include, but are not limited to, touch screens, PDAs, and cell phones. Haptic interaction refers to both manual input to a device and haptic feedback provided by the device. With sensing technologies, mobile devices can receive manual inputs through pressure-sensitive touch screens with or without individual keys. Most mobile devices also provide touch feedback in the form of vibration alerts, a useful and discreet feature especially when the device is in silent mode. At the same time, keyboards on mobile devices are disappearing to make room for larger display screens and thinner profiles, yet many people find it disconcerting to type on a surface with only visual but no haptic feedback. As touch screen technology gains popularity, the need has risen for key-click feedback signals that serve as confirmation of key presses, especially when the user's eyes are busy with other tasks. The idea of "active click" was first introduced by Fukumoto and Sugimura [2] where a vibrotactile actuator attached to the back of a touch panel generated a click-like vibrating pulse whenever the screen was tapped by a finger. Since then, several technologies have been implemented to generate haptic feedback. For example, Chang and O'Sullivan used a multifunction transducer [3]; Brewster's group adopted C2 tactors in

- H.-Y. Chen is with the Motorola Corporation, 600 US Hwy 45, Libertyville, IL 60048. E-mail: nelly.hychen@gmail.com.

- J. Park and H.Z. Tan are with the Haptic Interface Research Laboratory at Purdue University, 465 Northwestern Avenue, West Lafayette, IN 47907. E-mail: \{park183, hongtan\}@purdue.edu.

- S. Dai is with the Materials Science and Engineering Center, Sandia National Laboratories, PO Box 5800, MS 0959, Albuquerque, NM 87185. E-mail: sxdai@sandia.gov.

Manuscript received 20 July 2010; revised 29 Mar. 2011; accepted 12 Apr. 2011; published online 16 May 2011.

Recommended for acceptance by $\mathrm{H}$. Kajimoto.

For information on obtaining reprints of this article, please send e-mail to: toh@computer.org, and reference IEEECS Log Number TH-2010-07-0037.

Digital Object Identifier no. 10.1109/ToH.2011.21. most of their studies [4], [5]; and Lee et al. utilized a solenoid actuator to create feedback for a stylus [6]. In addition, piezoelectric actuators have been used in several applications involving handheld mobile devices [7], [8], [9], [10], [11], [12], [13], [14].

The present study focuses on the design and evaluation of haptic signals generated by a piezoelectric actuator that emulates key-click sensations. Our study is different from previous research on "tacton" [15] or "haptic icons" [16] in that we focus on signals that emulate key clicks, as opposed to any vibrotactile signals that may make up a tactile vocabulary. Our efforts also differ from those of earlier work on haptic devices for sensory substitution (e.g., [17]; see also [18] for a review) in that we use signals with an intuitive meaning (key clicks) instead of abstract signals that require extensive user training of a specific coding scheme (e.g., tactile aids for individuals with hearing impairments). Finally, instead of searching for signals that feel "pleasant" (e.g., [7]), our aim is to design a set of identifiable signals that can be used with different functions on a mobile device. We use the term "identifiable signals" to refer to a set of distinctive stimulus alternatives that can be easily identified in isolation (i.e., identification) as opposed to in comparison with other stimuli (i.e., discrimination). Instead of a relatively high level of identification (e.g., above 80 percent correct), we aimed to reach a near 100 percent identification accuracy. In many applications such as a belt with haptic waypoint information [19], a vest displaying haptic communication signals [20], or a mobile device with haptic alerts [15], recognition accuracy needs to be near perfect instead of being just "good enough" because the cost of misidentification can be high. In mobile applications, identifiable key clicks can enable eye-free operations when the user is unable to look at or see the device.

The problem of designing a set of perceptually identifiable signals for a given actuator has been studied in various contexts in the past. The common theme is to map the physical 
parameter values for driving the actuator to perception, so that different sensations can be achieved by judicious selections of these parameter values. These identifiable signals can then be encoded for a specific application. For example, Geldard [21] studied the use of vibrotactile frequency, amplitude, duration, locus in space, and wave complexity in encoding speech elements with an array of tactors. After discarding frequency (for interacting with intensity perception) and wave complexity (for being nondiscriminable), a total of 45 (3 amplitudes $\times 3$ durations $\times 5$ loci on the chest) vibrotactile signals were created, each representing an English alphabet letter, a single-digit number, or a short word (e.g., "of," "the," "in," "and"). One participant was able to receive 38 words per minute with this system. Maclean and colleagues have conducted extensive studies characterizing the relation between physical parameters and perceptual dimensions using a multidimensional scaling technique (MDS) [11], [16], [22]. In [22], these researchers used the MDS solution space to demonstrate and measure the perceptual distinctiveness of periodic vibrotactile signals varying in its waveform. In another study on the design of the tactile equivalent of ring tones for mobile devices, Brown et al. [15] mapped location, rhythm, and roughness parameters to vibrotactile alerting signals to indicate time-until-appointment $(30,15,5 \mathrm{~min})$, type of alert (meeting, lecture, tutorial), and importance (low, medium, high), respectively. Performance in terms of percent-correct scores and information transfer (IT) were reported.

The present study addresses similar questions as these previous investigations: What are the key physical parameters that affect perception in a predictable way? For each parameter, how many levels can be correctly identified without error? When multiple parameters vary in a signal set, by how much does the identification of each parameter level deteriorate? More importantly, how to reduce the number of levels per parameter so that identification of signals varying in multiple parameters remains perfect?

Various methods have been employed to study these questions. In Geldard's work [21], discrimination experiments were carried out to measure the just noticeable difference (JND) within each parameter range. The results indicated that there were about 15-17 JNDs for amplitude over a reference amplitude range of 20 to 400 microns, and 25 JNDs for duration over a reference duration range of 0.1 to $2.0 \mathrm{~s}$. With no further explanation, the author claimed that for practical purposes, three levels of amplitude, three levels of duration, and five loci on the chest could be included in the signal set. The selection of the parameter levels would have been more convincing if the author had conducted absolute identification experiments to measure the maximum number of identifiable signals, or the "channel capacity," of each parameter [23]. MacLean and colleagues [11], [16], [22] used the MDS technique to discover the perceptual dimensions associated with physical parameters. This technique has also been applied to the study of haptic texture perception [24] and perfumers' odor perception space [25]. One of the main difficulties in conducting an MDS experiment is the amount of time required to collect scaling data for all pairs of stimuli. A cluster-sorting method was proposed in [16] to speed up data collection, although there remain some unresolved issues and concerns with this approach [26]. In Brown et al.'s work [15], performance was reported in terms of percent-correct scores and information transfer. The former can be misleading in the sense that a decrease in percent-correct score with a larger stimulus set does not necessarily imply that the total number of identifiable signals have decreased. Although these authors also report information transfer, it was used mainly as a performance metric rather than an integral part of the stimulus design process.

The present study uses an information theoretical framework to study the design and evaluation of identifiable keyclick signals for mobile devices. One-dimensional and multidimensional absolute identification experiments are conducted to measure the channel capacity associated with a single physical parameter and multiple parameters, respectively. In one-dimensional absolute identification experiments, the values of the background parameters (i.e., the nontarget physical parameters making up a stimulus) are either kept fixed or varied randomly. While the fixed-background experiments allow us to estimate the "ideal" channel capacity achievable with a single physical parameter, the roving-background experiments produce a more "realistic" channel capacity when multiple parameters must be attended to in order to identify a signal [27]. We show how these experiments can guide the design of a multivariable stimulus set, and under what conditions results from one-dimensional absolute identification experiments can be used to predict the outcome of a multidimensional absolute identification experiment, the latter of which is usually too time-consuming to conduct for practical purposes.

The present study makes two important contributions. From a methodology perspective, we demonstrate how to assess the overall information transmission capacity of a stimulus set with multiple parameters that interact perceptually. From an application perspective, we provide the specifications for a set of identifiable key-click simulation signals that can be incorporated into mobile devices equipped with piezoelectric actuators. In what follows, we summarize the general methods in Section 2. Details specific to the four experiments conducted during the present study are presented in Sections 3 to 5 . The paper concludes in Section 6.

\section{General Methods}

\subsection{Apparatus}

The test apparatus resembled a typical mobile phone in its size and appearance (see Fig. 1). A single layer piezoelectric actuator (CTS standard 3,203, $4 \mathrm{~cm} \mathrm{~L} \times 3.5 \mathrm{~cm} \mathrm{~W} \times 0.2 \mathrm{~mm}$ $\mathrm{H}, 147 \mathrm{nF}$ capacitance, occupying the lower half of the apparatus) was affixed to a stainless steel plate that served as the cover of the apparatus. A piece of polycarbonate frame at the same size as the stainless steel plate was attached to the back of the apparatus. Four force sensing resistors (FSRs from Interlink) were mounted at the corners of the intended keypad area and sandwiched between the polycarbonate frame and a polycarbonate back plate. They were used to trigger a high-voltage input pulse to the piezo whenever the total force exceeded $200 \mathrm{~g}$ (or equivalently, a resistance of 


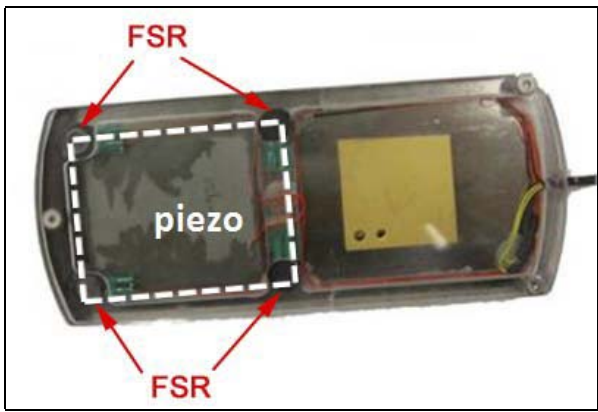

(a) Back view

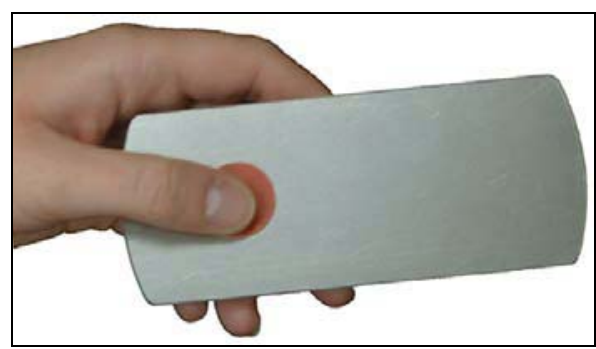

(b) Front view

Fig. 1. (a) Back view as seen through a clear Plexiglas cover and (b) front view of test apparatus. (From [1], Fig. 1, (C) 2010 IEEE).

$20 \mathrm{k} \Omega$ for the four FSRs connected in parallel). ${ }^{1}$ The $200 \mathrm{~g}$ force value was selected empirically. To emulate the weight of a typical mobile phone, a piece of metal weighing $40 \mathrm{~g}$ was glued to the upper half of the apparatus (the yellow block in Fig. 1a). The total weight of the apparatus was about $78 \mathrm{~g}$. A red dot marked the center of the piezoelectric actuator where the participants were told to press down with the thumb and feel a virtual key click (see Fig. 1b). Upon detection of a key press through the FSRs, a waveform was sent through a computer sound card (Creative Sound Blaster SB0100, Creative Resource, Singapore) to a voltage amplifier with a gain of 100 (Dual Channel High Voltage Precision Power Amplifier, Model 2,350, TEGAM, Inc., Geneva, OH). The output of the amplifier was subsequently sent to the piezoelectric actuator to create the sensation of a virtual key click. ${ }^{2}$

\subsection{Participants}

Twelve participants (P1-P12; 3 females; all right-handed except P12; age range 23-43 years old) took part in the present study. Participants P1-P3 were research staff and experienced with haptic devices. They participated in all the experiments in the present study. Participants P4-P12 were compensated for their time. They completed the first three of the four experiments conducted in the present study. All participants signed a written consent form approved by the Institute Review Board at Purdue University.

1. The latency between the detection of a $>200 \mathrm{~g}$ force by the FSR and the onset of a key-click signal was less than $1 \mathrm{~ms}$ using a PC. In real applications where the latency is limited by firmware, the latency can be significantly longer (e.g., $\approx 40 \mathrm{~ms}$ in Motorola's ROKR E8 music phone).

2. The haptic stimuli could be sensed by all the fingers holding the test apparatus. However, the signal was the strongest near the center of the piezoelectric actuator, and all participants (including the authors) located the key-click feedback signal at where the thumb was. It only became apparent that the signal could be felt by the four fingers holding the test apparatus if the thumb was lifted away and someone else pressed on the red dot to trigger the haptic stimuli.

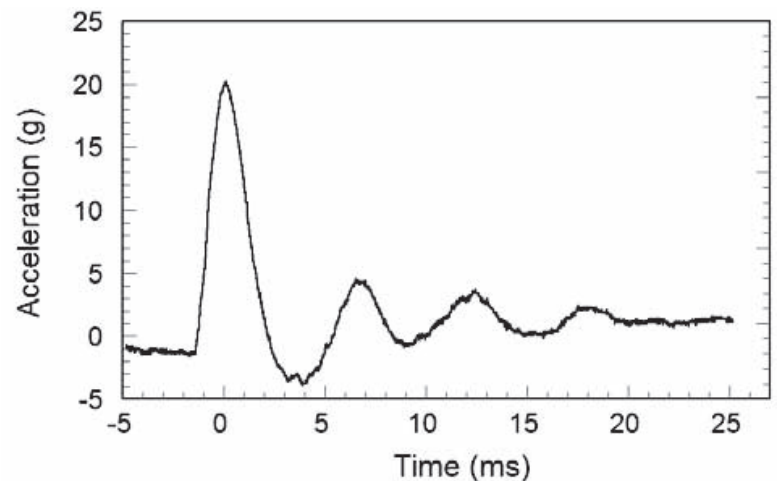

(a) Acceleration profile of a telephone key. The $0 \mathrm{~ms}$ time on the abscissa is arbitrarily aligned with the first peak.

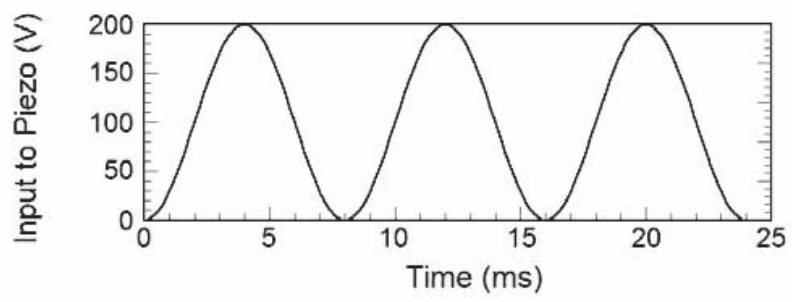

(b) A full-scale waveform for driving the piezoelectric actuator

Fig. 2. (a) Recorded acceleration profile of a key press; and (b) a typical input waveform for driving the piezoelectric actuator.

\subsection{Stimuli}

To determine the shape of waveforms for driving the piezoelectric actuator, acceleration profiles of pop-dome keys on a telephone, a computer keyboard, and a cell phone were measured. Fig. 2a shows a typical recording from the keypad of an office phone during the key-down phase. There is a clear initial pulse, followed by several "ringing" pulses with diminishing amplitudes. Based on the measurements, raised sinusoidal waveforms were used to drive the piezoelectric actuator (see Fig. $2 b$ ).

A series of preliminary experiments were conducted to determine the relevant parameters for generating key-click signals on the piezoelectric actuator. Among the variables considered were peak amplitude, frequency, number of cycles, initial/peak velocity, and initial/peak acceleration. Measurements were also taken to examine the transfer function of the piezoelectric actuator. In the interest of space, readers are referred to the [Appendices, 28] for details. In the end, three parameters were found to influence the perceived quality of simulated key clicks: amplitude, frequency, and number of cycles of the sinusoidal waveform. Amplitude of the waveform contributed to the overall perceived intensity of a key-click signal. The maximum amplitude was $200 \mathrm{~V}$ using the setup described in Section 2.1. Frequency of the waveform determined the perceived "crispness" of a key-click signal. The frequency range was selected to be $125-500 \mathrm{~Hz}$ that corresponded to perceptually "dull" to "crisp" key clicks. Finally, the number of cycles also contributed to the perceived intensity of the signal, but more than three cycles resulted in an eerie sensation of something alive. Therefore, the number of cycles ranged from 1 to 3 in the present study. Other waveforms such as sinusoidal pulses 
TABLE 1

The Full Stimulus Set Used in Experiments I and II

\begin{tabular}{|c|c|c|c|c|c|}
\hline \multirow{2}{*}{ Amplitude } & A1 & $\mathrm{A} 2$ & A3 & A4 & A5 \\
\hline & $40 \mathrm{~V}$ & $80 \mathrm{~V}$ & $120 \mathrm{~V}$ & $160 \mathrm{~V}$ & $200 \mathrm{~V}$ \\
\hline \multirow{2}{*}{ Frequency } & F1 & F2 & F3 & F4 & \\
\hline & $125 \mathrm{~Hz}$ & $250 \mathrm{~Hz}$ & $375 \mathrm{~Hz}$ & $500 \mathrm{~Hz}$ & \\
\hline \multirow{2}{*}{$\begin{array}{c}\text { Number of } \\
\text { Cycles }\end{array}$} & $\mathrm{C} 1$ & C2 & $\mathrm{C} 3$ & & \\
\hline & 1 & 2 & 3 & & \\
\hline
\end{tabular}

with exponentially decaying envelopes were also investigated, but were found not to result in perceptually distinct key-click sensations when compared to the signal shown in Fig. $2 b$.

The full stimulus set used in Experiments I and II of the present study consisted of 60 alternatives ( 5 amplitude $\times$ 4 frequency $\times 3$ number of cycles). Table 1 lists the values for the three parameters and their associated labels. The highlighted values indicate the parameter values when the corresponding parameter was fixed as a background parameter (see Section 2.4.2). This full stimulus set was pared down in Experiments III and IV for reasons that will become clear later. The proximal stimuli were characterized by an accelerometer placed near the center of the piezo actuator. Fig. 3 compares the PC output waveform to the measured piezo acceleration profile for two representative signals.

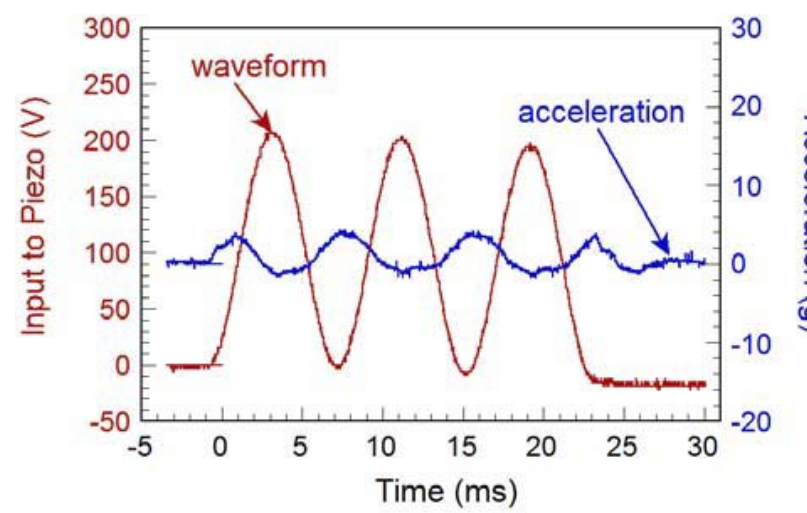

(a)

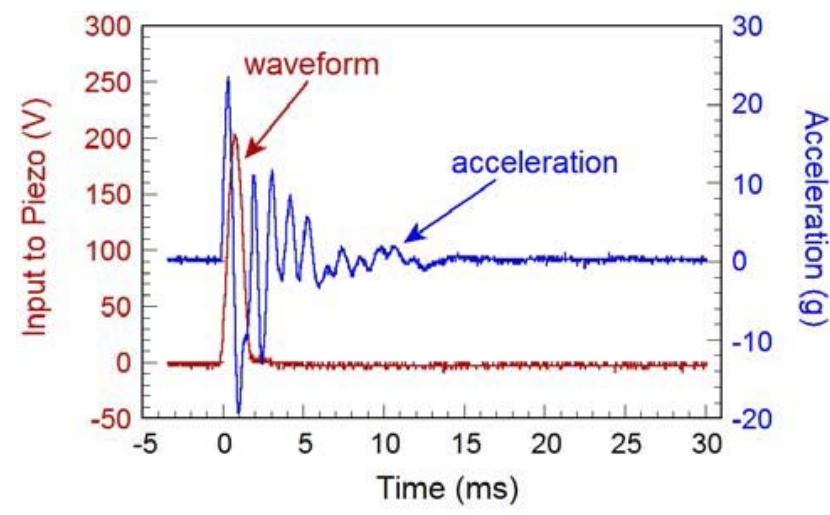

(b)

Fig. 3. Comparison of input waveform to piezo and measured piezo acceleration profile for two stimuli. (a) A5 $=200 \mathrm{~V}, \mathrm{~F} 1=125 \mathrm{~Hz}$, $\mathrm{C} 3=3$ cycles. (b) $\mathrm{A} 5=200 \mathrm{~V}, \mathrm{~F} 4=500 \mathrm{~Hz}, \mathrm{C} 1=1$ cycle. (Modified from [1], Figs. 2 and 3, (c) 2010 IEEE)

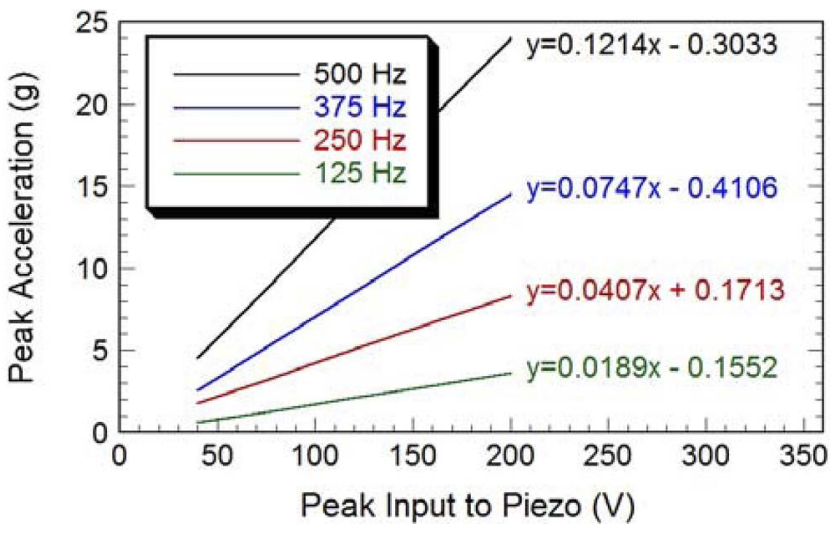

Fig. 4. Calibration curve for the piezoelectric actuator.

To characterize the proximal stimuli in response to the stimuli listed in Table 1, the pizeoactuator responses were calibrated in terms of the peak acceleration at the red dot (Fig. 1b) as a function of the peak voltage of one cycle of a raised cosine input waveform. The results are shown in Fig. 4.

\subsection{Procedures}

In this section, we first describe the procedures for running an absolute identification experiment, which are used in all four experiments conducted in the present study. We then discuss the distinction between a one-dimensional (1D) and a multidimensional (multi-D) identification experiment. This is followed by a presentation of the procedures for running 1D identification experiments with fixed or roving background. We then discuss how the results from 1D and multi-D identification experiments may be related, in the form of a general additivity law for information transfer.

\subsubsection{Absolute Identification Experiment}

A typical identification experiment consists of the following steps: A set of $\mathrm{K}$ stimuli $\left(S_{i, 1} 1 \leq i \leq K\right)$ is constructed for the experiment; a set of $\mathrm{K}$ responses $\left(R_{j}, 1 \leq j \leq K\right)$ is constructed with a one-to-one association with each of the $\mathrm{K}$ stimuli; the participant is presented with stimuli selected at random from the stimulus set; on each presentation, the participant chooses a response from the response set; and the experimental results are tabulated in the form of a stimulusresponse confusion matrix from which measurements of information transfer are computed. The quantity IT measures the increase in information about the signal transmitted resulting from knowledge of the received signal. For a particular stimulus-response pair $\left(S_{i}, R_{j}\right)$, the quantity IT is given by $\log _{2}\left[P\left(S_{i} \mid R_{j}\right) \mid P\left(S_{i}\right)\right]$, where $P\left(S_{i} \mid R_{j}\right)$ is the conditional probability of $S_{i}$ given $R_{j}$, and $P\left(S_{i}\right)$ is the a priori probability of $S_{i}$. The average information transfer is given by

$$
I T=\sum_{j=1}^{K} \sum_{i=1}^{K} P\left(S_{i}, R_{j}\right) \log _{2}\left(\frac{P\left(S_{i} \mid R_{j}\right)}{P\left(S_{i}\right)}\right),
$$

or, equivalently

$$
I T=\sum_{j=1}^{K} \sum_{i=1}^{K} P\left(S_{i}, R_{j}\right) \log _{2}\left(\frac{P\left(S_{i}, R_{j}\right)}{P\left(S_{i}\right) P\left(R_{j}\right)}\right),
$$


where $P\left(S_{i}, R_{j}\right)$ is the joint probability of stimulus $S_{i}$ and response $R_{j}$, and $P\left(R_{j}\right)$ is the probability of $R_{j}$.

The maximum likelihood estimate of IT derived from a stimulus-response matrix, denoted $\mathrm{IT}_{\text {est }}$, is computed by approximating the underlying probabilities with frequencies of occurrence

$$
I T_{e s t}=\sum_{j=1}^{K} \sum_{i=1}^{K} \frac{n_{i j}}{n} \log _{2}\left(\frac{n_{i j} \cdot n}{n_{i} \cdot n_{j}}\right),
$$

where $n$ is the total number of trials collected, $n_{i j}$ is the number of times the joint event $\left(S_{i}, R_{j}\right)$ occurs, and $n_{i}=$ $\sum_{j=1}^{K} n_{i j}$ and $n_{j}=\sum_{i=1}^{K} n_{i j}$ are the row and column sums. A related measure, computed as $2^{\text {ITest }}$, is interpreted as the number of stimulus levels that can be correctly identified without error.

It has been shown that $\mathrm{IT}_{\text {est }}$ is a statistically biased estimated of IT and the bias generally decreases as total number of trials increases [29]. In the present study, we use Miller's recommendation that the total number of trials should be at least $5 \mathrm{~K}^{2}$, where $\mathrm{K}$ is the number of stimulus alternatives.

More details on information theory as it relates to psychophysical studies can be found in [23]. A summary of issues related to the design of an identification experiment can be found in [30].

\subsubsection{Identification Experiment with Fixed or Roving Background}

The stimulus set shown in Table 1 is called a multidimensional stimulus set in the sense that more than one parameter can vary in order to make up the stimulus alternatives. When running a 1D identification experiment, the parameter being identified is called the target, and the other parameters the background. For example, in a 1D amplitude identification experiment, amplitude is the target and frequency and number of cycles are the background. A multi-D identification experiment has more than one target parameters that need to be identified. For example, in a 3D identification experiment using the stimulus set shown in Table 1, all the three parameters are targets.

In the present study, we conducted 1D identification experiments with fixed or roving background. With fixed background, the background parameters are assigned fixed values throughout an experiment while the value of the target parameter is randomized from trial to trial. The IT results so obtained represent the best identification performance that can be achieved with the target parameter. In a 1D identification experiment with roving background, however, the values of all parameters are randomly selected from trial to trial. The participant is asked to identify the value of the target parameter while ignoring the random variations in the background parameters. The roving background procedure is more demanding than the fixed background procedure, and the resulting IT is usually lower. The lower IT values from identification experiments with roving background reflect the interactions among the parameters that make up the stimulus alternatives.

The participants wore earmuffs (Peltor, with a nominal sound reduction of $29 \mathrm{~dB}$ for noise levels up to $105 \mathrm{~dB}$ ) to block possible audio cues and noises. Each experimental run started with a short training session that lasted 5 to $15 \mathrm{~min}$. Participants could choose the level of the target parameter they would like to feel by pressing a number on the keyboard. The training session ended when the participants decided to start the experiment. Participants were instructed to press down on the red dot attached to the test apparatus (see Fig. 1b) to trigger the presentation of a key-click signal. Participants were asked to indicate the perceived level of the target parameter by typing the corresponding number on the computer keyboard. Trial-by-trial correct-answer feedback was provided. The total number of trials was divided into multiple runs. Between runs, participants were given the option to take a break if needed.

In Experiment I where fixed background was used, the values of the background parameters were fixed at A3 $(120 \mathrm{~V})$ for amplitude, F2 $(250 \mathrm{~Hz})$ for frequency, and C2 $(2$ cycles) for number of cycles (see the highlighted parameter values in Table 1). For example, in a 1D amplitude identification experiment with fixed background, the frequency was fixed at $250 \mathrm{~Hz}$ and the number of cycles was fixed at 2. The participant's task was to identify the level of the amplitude parameter when stimulus amplitude varied from trial to trial.

In Experiment II where roving background was used, the values of the three parameters were chosen independently and randomly from trial to trial. For example, in a 1D amplitude identification experiment with roving background, any of the 60 stimulus alternatives shown in Table 1 may be presented on a given trial. The participant's task was to identify the level of the amplitude parameter despite random variations in the frequency and number of cycles of the stimulus.

Experiments III and IV used a 3D identification paradigm. The main difference in the 3D experiment was that the participants had to identify all three parameters on each trial. In Experiment III, they were asked to identify the level of each parameter by sequentially entering three numbers that corresponded to amplitude, frequency, and number of cycles, respectively. In Experiment IV, the participants were asked to use a graphic code to identify the stimulus presented on each trial.

\subsubsection{A General Additivity Law}

With any multi-D stimulus set such as the one in the present study, it is generally of interest to measure the multi-D IT achievable with such a stimulus set. To run a full-scale 3D identification experiment with the 60 stimuli shown in Table 1 , however, would require a minimum of $5 \times 60^{2}=$ 18,000 trials!

Alternatively, one can ask the question of whether a multi-D IT can be predicted from the sum of 1D ITs estimated with each of the parameters making up the stimulus set. In general, IT(multi-D) $<\Sigma$ IT(1-D), due to perceptual interferences among the stimulus parameters which is generally not accounted for by 1D identification experiments with fixed background (e.g., [31]). However, it appeared that when 1D identification experiments were conducted with roving background, then the sum of 1-D ITs will approximate the multi-D IT closely [32]. Durlach et al. [27] proposed a general additivity law that predicted, in the case of the present study 


$$
\begin{aligned}
\mathrm{IT}(\mathrm{A}, \mathrm{F}, \mathrm{C}) \approx & \mathrm{IT}(\mathrm{A} \mid \operatorname{rovF}, \mathrm{C})+\mathrm{IT}(\mathrm{F} \mid \operatorname{rovA}, \mathrm{C}) \\
& +\mathrm{IT}(\mathrm{C} \mid \operatorname{rov} \mathrm{A}, \mathrm{F}),
\end{aligned}
$$

where IT(A, F, C) denotes the IT from a 3D identification experiment where all three parameters, the amplitude, frequency, and number of cycles of a key-click signal, have to be identified; IT(A $\mid$ rov F, C) is the 1D IT from an amplitude identification experiment with frequency and number of cycles as the roving background; IT(F I rov A, C) is the 1D IT from a frequency identification experiment with roving amplitude and number of cycles; and IT(C I rov A, F) is the 1D IT from an identification experiment with number of cycles as the target and amplitude and frequency as the roving background. The general additivity law therefore states that a multi-D IT can be predicted from the sum of 1D ITs, if the perceptual dependence of the parameters is properly accounted for by the roving background paradigm.

Since it takes many more trials to collect enough number of trials for a 3D identification experiment than those required of several 1D identification experiments, it generally takes less number of total trials to estimate all three terms on the right of (4) than to estimate the one term on the left of the equation. For example, in the case of the present study, a minimum of 18,000 trials are needed in order to obtain an unbiased IT estimate from a 3D identification experiment, as compared to a total of only 250 trials needed from three 1D identification experiments with roving background (i.e., $5 \times 5^{2}$ trials for amplitude identification, $5 \times 4^{2}$ trials for frequency identification, and $5 \times 3^{2}$ trials for the identification of number of cycles). Therefore, the general additivity law can significantly save the experimental time required to obtained unbiased estimates of multi-D ITs.

In the present study, we measured 1D ITs with fixed and roving background, and compared their, respectively, sums to the 3D IT obtained from a 3D identification experiment. To make it tractable to collect sufficient number of trials in the 3D identification experiment in order to obtain an unbiased estimate of IT, we used the results of the $1 \mathrm{D}$ identification experiments to pare down the number of alternatives in the full stimulus set shown in Table 1 . Therefore, our experiments were designed to guide the development of a final set of perceptually identifiable keyclick signals, and at the same time to verify the general additivity law proposed by Durlach et al. [27].

\subsection{Data Analysis}

Results from each experiment were summarized in a K-by-K stimulus-response confusion matrix. The $\mathrm{IT}_{\text {est }}$ values were also calculated using (3). Although we present one confusion matrix per experimental condition by pooling multiple participants' data, the $\mathrm{IT}_{\text {est }}$ values were always calculated for individual participants first and then averaged.

In addition to the 1D IT results for each stimulus parameter, denoted IT(A), IT(F), or IT(C), the sum of the three IT values were also reported. The sums, denoted IT(SUM), were calculated separately for fixed and roving background experiments. They were used to check the validity of the general additivity law proposed in [27].
TABLE 2

Pooled Data from Experiment I

\begin{tabular}{|c|c|c|c|c|c|c|}
\hline & \multicolumn{5}{|c|}{ Response } \\
\hline & & $\mathrm{A} 1$ & $\mathrm{~A} 2$ & A3 & $\mathrm{A} 4$ & A5 \\
\hline \multirow{5}{*}{$\begin{array}{l}\frac{\mathscr{D}}{\bar{J}} \\
\frac{\vec{E}}{\bar{D}}\end{array}$} & $\mathrm{~A} 1$ & 285 & 30 & 1 & 0 & 0 \\
\hline & $\mathrm{A} 2$ & 65 & 212 & 44 & 14 & 0 \\
\hline & A3 & 10 & 79 & 178 & 89 & 18 \\
\hline & A4 & 2 & 15 & 76 & 181 & 150 \\
\hline & A5 & 2 & 4 & 14 & 86 & 245 \\
\hline
\end{tabular}

(a) Identification of Amplitude with Fixed Background

\begin{tabular}{|c|c|c|c|c|c|}
\hline & \multicolumn{4}{|c|}{ Response } \\
\hline & & F1 & $\mathrm{F} 2$ & F3 & $\mathrm{F} 4$ \\
\hline \multirow{4}{*}{ 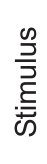 } & F1 & 256 & 16 & 8 & 1 \\
\hline & $\mathrm{F} 2$ & 9 & 141 & 94 & 43 \\
\hline & F3 & 4 & 89 & 125 & 103 \\
\hline & $\mathrm{F} 4$ & 14 & 47 & 67 & 183 \\
\hline
\end{tabular}

(b) Identification of Frequency with Fixed Background

(c) Identification of Number of Cycles with Fixed Background

\begin{tabular}{cc|ccc}
\hline \multirow{4}{*}{ Stimulus } & & \multicolumn{3}{|c}{ Response } \\
& & C1 & C2 & C3 \\
\hline & C2 & 186 & 23 & 3 \\
& C3 & 3 & 121 & 43 \\
\hline
\end{tabular}

\section{EXPERIMENTS I \& II: 1D IDENTIFICATION EXPERIMENTS WITH FIXED AND ROVING BACKGROUND}

In the first two experiments, the 1D IT achievable with the parameters amplitude, frequency, and number of cycles were estimated, using both a fixed background (Experiment I) and a roving background (Experiment II) paradigm.

\subsection{Methods}

As explained in Section 2.3, the amplitude parameter in the stimulus set used in the present study had five levels, the frequency parameter four levels, and the number of cycles parameter three levels. Accordingly, the total number of trials collected during the $1 \mathrm{D}$ identification experiments was different for each of the parameters. In order to obtain unbiased IT estimates, a minimum of 125 trials was needed for a 1D amplitude identification experiment, 80 trials for frequency identification, and 45 trials for the identification of number of cycles. Since we divided all experiments into 50-trial runs, a total of 3 runs (150 trials) were collected for 1D amplitude identification experiments, 2 runs (100 trials) for frequency identification, and 1 run (50 trials) for identification of number of cycles. In all, a total of 12 50trial runs were conducted per participant ( 6 for the fixed background condition, and another 6 for the roving background condition). It took each participant between 1 to 2 hours to complete the experiments, including the time for breaks.

\subsection{Results}

Table 2 shows the stimulus-response confusion matrices pooled from all participants for the fixed-background 
TABLE 3

Pooled Data from Experiment II

(a) Identification of Amplitude with Roving Background

\begin{tabular}{|c|c|c|c|c|c|c|}
\hline & \multicolumn{5}{|c|}{ Response } \\
\hline & & A1 & A2 & A3 & A4 & A5 \\
\hline \multirow{5}{*}{ 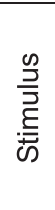 } & A1 & 278 & 63 & 14 & 2 & 0 \\
\hline & A2 & 113 & 141 & 86 & 23 & 5 \\
\hline & A3 & 35 & 87 & 120 & 97 & 42 \\
\hline & A4 & 8 & 28 & 111 & 106 & 109 \\
\hline & A5 & 2 & 20 & 57 & 95 & 158 \\
\hline
\end{tabular}

(b) Identification of Frequency with Roving Background

\begin{tabular}{|c|c|c|c|c|c|}
\hline & \multicolumn{4}{|c|}{ Response } \\
\hline & & F1 & F2 & F3 & F4 \\
\hline \multirow{4}{*}{ 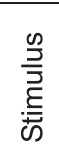 } & F1 & 222 & 41 & 20 & 15 \\
\hline & $\mathrm{F} 2$ & 47 & 106 & 104 & 69 \\
\hline & F3 & 34 & 52 & 104 & 100 \\
\hline & F4 & 17 & 49 & 88 & 132 \\
\hline
\end{tabular}

(c) Identification of Number of Cycles with Roving Background

\begin{tabular}{cc|ccc}
\hline & & \multicolumn{3}{|c}{ Response } \\
& & C1 & C2 & C3 \\
\hline \multirow{3}{*}{ Stimulus } & C1 & 108 & 63 & 18 \\
& C2 & 69 & 72 & 55 \\
& C3 & 53 & 61 & $\mathbf{1 0 1}$ \\
\hline
\end{tabular}

experiments (Experiment I). Each cell entry shows the number of trials that a particular stimulus was called a particular response. The shaded cells along the main diagonals of the confusion matrices correspond to the number of trials with correct answers. Overall, the percentcorrect score for each stimulus ranged from 43 (A4) to 90 percent (A1) for amplitude (Table 2a), 39 (F3) to 91 percent (F1) for frequency (Table 2b), and 63 (C2) to 88 percent (C1) for number of cycles (Table 2c). It appears that the lowest stimulus level always resulted in the highest identification accuracy. Looking at the error patterns in Tables $2 \mathrm{a}$ and 2c, the majority of confusions occurred between adjacent parameter levels (i.e., most of the error trials occurred among the cells adjacent to the main diagonal cells).

Table 3 shows the stimulus-response confusion matrices pooled from all participants for the roving-background experiments (Experiment II). As expected, the percentcorrect scores for each stimulus were generally lower than those in the 1D identification experiment with fixed background (Experiment I). The percent-correct scores ranged from 29 (A4) to 78 percent (A1) for amplitude (Table 3a), 33 (F2) to 74 percent (F1) for frequency (Table 3b), and 37 (C2) to 57 percent (C1) for number of cycles (Table 3c). Again, the highest identification accuracy was associated with the lowest stimulus level for each parameter. Compared to the data from the fixed background experiments (Table 2), there were significantly more error trials in the roving background experiments, and errors were more widely spread away from the main diagonal cells. For instance, A2 was misidentified as A1 113 times with roving background (as compared to 65 times with fixed background); F2 was misidentified as F1 47 times with roving background (as
TABLE 4

Information Transfer (in Bits) from Experiments I \& II

(a) 1-D IT Values with Fixed Background

\begin{tabular}{c|ccc|c}
\hline Participant & IT(A) & IT(F) & IT(C) & IT(SUM) \\
\hline P1 & 1.37 & 1.07 & 1.24 & 3.68 \\
P2 & 1.19 & 1.10 & 1.18 & 3.47 \\
P3 & 1.35 & 1.19 & 1.56 & 4.10 \\
P4 & 1.15 & 1.08 & 0.88 & 3.11 \\
P5 & 0.79 & 0.63 & 0.48 & 1.90 \\
P6 & 0.76 & 0.19 & 0.45 & 1.40 \\
P7 & 1.38 & 0.74 & 1.07 & 3.19 \\
P8 & 1.05 & 0.82 & 0.47 & 2.34 \\
P9 & 1.10 & 0.67 & 0.85 & 2.62 \\
P10 & 1.03 & 0.96 & 0.83 & 2.82 \\
P11 & 0.66 & 0.73 & 0.49 & 1.88 \\
P12 & 0.78 & 0.56 & 0.87 & 2.21 \\
\hline Average & $\mathbf{1 . 0 5}$ & $\mathbf{0 . 8 1}$ & $\mathbf{0 . 8 6}$ & $\mathbf{2 . 7 3}$ \\
STD & 0.25 & 0.29 & 0.35 & 0.81 \\
\hline
\end{tabular}

(b) 1-D IT Values with Roving Background

\begin{tabular}{c|ccc|c}
\hline Participant & IT(A) & IT(F) & IT(C) & IT(SUM) \\
\hline P1 & 0.54 & 0.68 & 0.24 & 1.46 \\
P2 & 0.51 & 0.76 & 0.26 & 1.53 \\
P3 & 0.66 & 1.03 & 0.10 & 1.79 \\
P4 & 0.71 & 0.32 & 0.36 & 1.39 \\
P5 & 0.54 & 0.14 & 0.14 & 0.82 \\
P6 & 0.51 & 0.13 & 0.17 & 0.81 \\
P7 & 0.83 & 0.31 & 0.21 & 1.35 \\
P8 & 0.60 & 0.45 & 0.05 & 1.10 \\
P9 & 0.92 & 0.50 & 0.36 & 1.78 \\
P10 & 0.59 & 0.53 & 0.14 & 1.26 \\
P11 & 0.67 & 0.15 & 0.10 & 0.92 \\
P12 & 0.66 & 0.52 & 0.04 & 1.22 \\
\hline Average & $\mathbf{0 . 6 5}$ & $\mathbf{0 . 4 6}$ & $\mathbf{0 . 1 8}$ & $\mathbf{1 . 2 9}$ \\
STD & 0.13 & 0.27 & 0.11 & 0.33 \\
\hline
\end{tabular}

compared to 9 times with fixed background); and C2 was misidentified as C1 69 times (as compared to 29 times with fixed background). Moreover, confusions occurred not only between adjacent parameter levels but also between stimuli that were two or more levels apart. For example, A1 was called A3 14 times with roving background (as compared to only 1 time with fixed background), and F2 was called F4 69 times with roving background (as compared to 43 times with fixed background).

Table 4 lists the IT values for all participants along with averages and standard deviations. The IT (SUM) columns report the summation of the 1DITs from the three parameters. In Experiment I with fixed background (Table 4a), P1-P3 achieved higher IT values than the rest of the participants, presumably because they were more familiar with the stimuli. The average 1D IT was 1.05 bits (2.07 items) for amplitude, 0.81 bit (1.75 items) for frequency, and 0.86 bit (1.82 items) for number of cycles across all 12 participants. The results indicate that the participants could correctly identify two amplitude levels $\left(2^{1.05-b i t s}=2.07\right.$ items $)$, but not quite two levels for frequency $\left(2^{0.81-b i t}=1.75\right.$ items $)$ or number of cycles $\left(2^{0.86-b i t}=1.82\right.$ items). The sum of the 1D ITs with fixed background was 2.73 bits $\left(2^{2.73-b i t s}=6.63\right.$ items), predicting that the participants should be able to correctly identify between 6 to 7 key-click signals varying in amplitude, frequency, and number of cycles. 
In Experiment II with roving background (Table 4b), P1P3 again achieved higher IT values than the rest of the participants, except for $\mathrm{P} 9$ who performed especially well in amplitude identification. The average 1D IT was 0.65 bit (1.57 items) for amplitude, 0.46 bit (1.38 items) for frequency, and 0.18 bit (1.13 items) for number of cycles. These results indicate that the participants could only correctly identify one level of amplitude, frequency, or number of cycles. The sum of the 1D ITs with roving background was 1.29 bits (2.45 items), predicting a dismal total of two perceptually identifiable key-click signals.

\subsection{Discussions}

The fixed-background experiments showed that information transfers for the experienced participants (P1-P3) were much higher than those for the inexperienced participants. The experienced participants could identify two levels of amplitude, two levels of frequency, and two to three levels for number of cycles. Participant P3 was able to identify the number of cycles perfectly. Of the remaining participants $\mathrm{P} 4-\mathrm{P} 12$, three of them (P5, P6, and P11) reached much lower ITs than the rest. During postexperimental debriefing, these participants commented that the tactile experience was unexpected and they probably did not spend enough time on training before proceeding to data collection. It thus appears that the participants received different amount of training and their performance could probably have been improved with more exposure to the key-click signals.

From the 1D fixed-background experiment to the $1 \mathrm{D}$ roving-background experiment, the sum of information transfers degraded from 2.7 (6.5 items) to 1.3 bits ( 2.5 items). The single-parameter IT dropped most noticeably in the roving-background experiment when number of cycles was the target parameter. This was due to the fact that both the amplitude and the number of cycles contributed to the perceived intensity of the key-click signals. With the fixedbackground paradigm, it was possible for the participants to attribute the changes in perceived intensity solely to the number of cycles. With the roving-background paradigm, however, it became difficult to ascertain whether a change in the overall perceived intensity was due to a change in the signal amplitude, the number of cycles, or both. Furthermore, it was especially difficult to identify the number of cycles when the signal amplitude was low. When the amplitude was high, it was easier to identify the number of cycles from a pulsating sensation, especially when the signal frequency was low (i.e., the period of each cycle was long).

While amplitude and frequency identification were equally easy for some participants in terms of similar ITs (e.g., P1-P5 and P10-P12), the amplitude cue was more dominant for other participants (P6-P9). Some participants found it hard to associate frequency with the perception of key clicks. They commented noticing only the overall perceived intensity of the stimuli, especially when the amplitude was low.

To investigate the predictive power of the IT(SUM) from the fixed-background and roving-background experiments, a 3D identification experiment was performed where all three parameters (amplitude, frequency, number of cycles) had to be identified simultaneously while their levels varied from trial to trial.
TABLE 5

Stimulus Set for Experiment III

\begin{tabular}{|ccc|c|}
\hline \multirow{2}{*}{ Amplitude } & $\mathrm{A} 1$ & $\mathrm{~A} 2$ & $\mathrm{~A} 3$ \\
\cline { 2 - 3 } & $40 \mathrm{~V}$ & $120 \mathrm{~V}$ & $200 \mathrm{~V}$ \\
\hline \multirow{2}{*}{ Frequency } & $\mathrm{F} 1$ & $\mathrm{~F} 2$ & \\
\cline { 2 - 3 } & $125 \mathrm{~Hz}$ & $500 \mathrm{~Hz}$ & \\
\hline $\begin{array}{c}\text { Number of } \\
\text { Cycles }\end{array}$ & $\mathrm{C} 1$ & $\mathrm{C} 2$ & \\
\cline { 2 - 3 } & 1 & 3 & \\
\hline
\end{tabular}

\section{EXPERIMENT III: 3D IDENTIFICATION EXPERIMENT}

\subsection{Methods}

As mentioned earlier in Section 2.4.3, the full stimulus set shown in Table 1 was pared down in terms of the number of levels per parameter in order to reduce the total number of stimulus alternatives in the 3D identification experiment, which in turn significantly reduced the total number of trials needed in order to obtain an unbiased estimate of IT(3D). Based on the results from Experiment I (1D identification experiment with fixed background), participants could on average identify 2.1 amplitude levels, 1.7 frequency levels, and 1.9 levels of number of cycles. With roving background (Experiment II), the number of levels reduced to 1.5 for amplitude, 1.4 for frequency, and 1.1 for number of cycles. To minimize the total number of trials needed in the 3D identification experiment, yet still ensure that identification performance would not be limited by the total number of stimulus alternatives, the number of levels per parameter was reduced to be just above the participants' performance levels in Experiment I. Specifically, there were three levels for the amplitude parameter $(40,120$, and $200 \mathrm{~V})$, two levels for the frequency parameter $(125$ and $500 \mathrm{~Hz})$, and two levels for number of cycles ( 1 cycle and 3 cycles). This resulted in a total of $12(3 \times 2 \times 2)$ stimulus alternatives in the 3D stimulus set, as shown in Table 5. Note that the labels for the parameter levels shown in Table 5 were different than those in Table 1, in the sense that A2 meant $80 \mathrm{~V}$ in Table 1 but $120 \mathrm{~V}$ in Table 5 .

The experimental procedure was essentially the same as those in Experiment II, except for the way the responses were entered. The participants were asked to respond to the perceived level of amplitude, frequency, and number of cycles, respectively, by entering three numbers in that order. For example, the correct response to the stimulus A3F1C2 would be " 3 ," " 1 ," and " 2 " entered that the sequence. For the total number of trials, a minimum of 720 trials were needed in order to obtain an unbiased IT (3D) for the 12 stimulus alternatives. Accordingly, a total of 750 trials, divided into 15 50-trial runs, were conducted. It took each participant between 30 to $90 \mathrm{~min}$ to complete the experiment.

\subsection{Results}

The results are shown in Table 6 in a 12-by-12 stimulusresponse confusion matrix with data pooled from all 12 participants. The percent-correct score for each stimulus ranged from 11 percent (A3F1C1) to 65 percent (A1F1C1). Certain stimulus pairs were more confused than the others. For instance, stimulus A1F2C1 was misidentified as A1F1C1 165 times (underlined in Table 6) indicating that when the 
TABLE 6

Pooled Data from Experiment III (3D Identification Experiment)

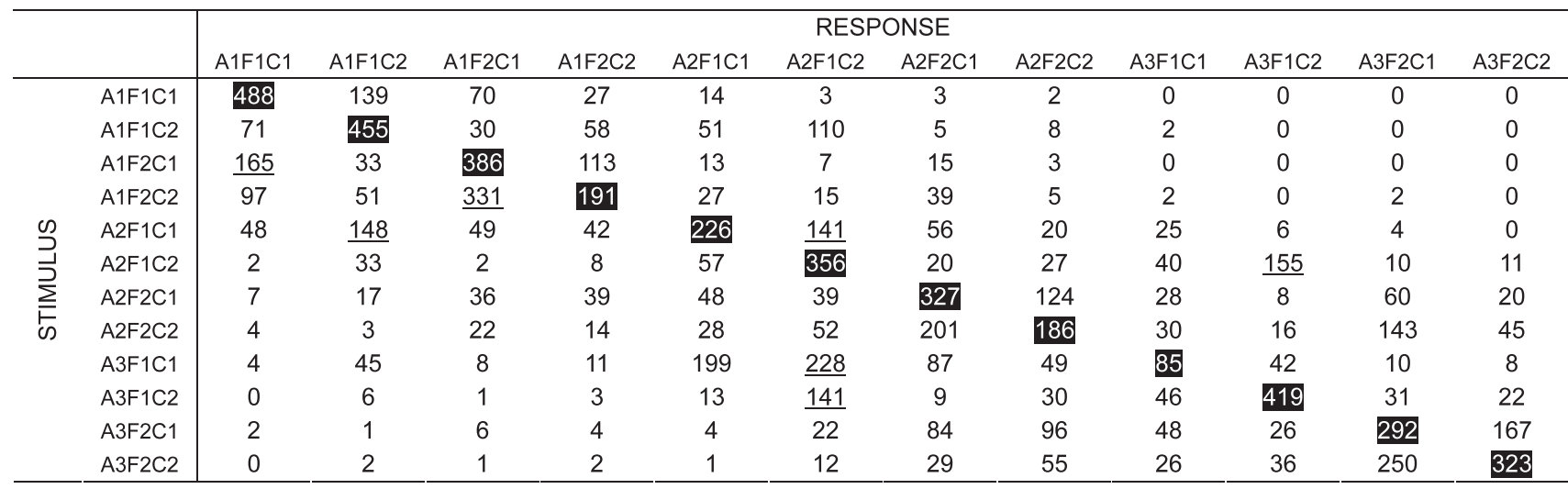

overall signal amplitude was low (A1), it was difficult to judge the frequency (crispness) of the signal. Signal A1F2C2 was misidentified as A1F2C1 331 times, indicating that it might also be difficult to judge the number of cycles when the signal amplitude was low. It was also observed that while both amplitude and number of cycles contributed to the perceived intensity of a signal, it was difficult to separate the contributions from each parameter. For example, signal A2F1C1 was misrecognized as A1F1C2 148 times and as A2F1C2 141 times; and signals A2F1C2 and A3F1C2 were misidentified as each other 155 and 141 times, respectively.

The IT (3D) calculated from Table 6 is 1.2 bits, indicating that the participants were able to identify only 2 out of the 12 signals perfectly. As it will become apparent later (see results of Experiment IV in Section 5), this is an underestimate of the participants' ability to identify key-click signals.

Table 7 shows the ITs for all participants along with the averages and standard deviations. The quantities IT_A, IT_F, and IT_C refer to the conditional ITs for amplitude, frequency, and number of cycles, respectively, that were calculated from the confusion matrix shown in Table 6 . The conditional ITs were calculated by collapsing the 12-by-12 confusion matrix along specific rows and/or columns. For instance, to obtain the conditional IT for amplitude, IT_A, the 12-by-12 confusion matrix was collapsed into a 3-by-3 matrix according to the amplitude levels. Regardless of the level of frequency or number of cycles, if the stimulus had A1 for its amplitude parameter and the participants' response contained $\mathrm{A} 1$, then the entries were added to the $(1,1)$ cell in the 3-by-3 confusion matrix. Likewise, if the stimulus had A1 and the response contained A3, then the entries were added to cell $(1,3)$, etc. The conditional IT for frequency and number of cycles were calculated in the same fashion. The quantities IT_A, IT_F, and IT_C therefore indicated the amount of information transmitted through each of the three parameters. From Table 7, the highest IT_A score was 0.83 bit (P7), the highest IT_F scores was 0.75 bit (P7), and the highest IT_C scores was 0.28 bit (P2).

\subsection{Discussions}

The conditional IT values (Table 7) can be compared with the ITs obtained on the same target parameters from 1D fixedand roving-background experiments (Table 4). First, it is noted that the average IT_A (0.63 bit), IT_F (0.40 bit), and IT_C ( 0.07 bit) values from the 3D identification experiment are much closer to their counterparts from the $1 \mathrm{D}$ rovingbackground identification experiment: $\operatorname{IT}(\mathrm{A})=0.65$ bit, $\operatorname{IT}(\mathrm{F})=0.46$ bit, and $\operatorname{IT}(\mathrm{C})=0.18$ bit (Table $4 \mathrm{~b})$. In comparison, the corresponding values from the 1D fixed-background identification experiment were much higher: $\operatorname{IT}(\mathrm{A})=1.05$ bits, $\operatorname{IT}(\mathrm{F})=0.81$ bit, and $\operatorname{IT}(\mathrm{C})=0.86$ bit (Table 4a). These results appear to support the general additivity law ((4) and [27]) in that the 1D ITs from the roving-background experiment match the $3 \mathrm{D}$ conditional ITs. Consequently, the sum of the 1D ITs, IT (SUM) in Table $4 \mathrm{~b}$, closely predicts the total IT from the 3D experiment, IT(3D) in Table 7. Second, for all participants except P10, the highest conditional IT was obtained with the amplitude parameter in the 3D identification experiment (see Table 7). It showed once again that participants tended to notice the overall perceived intensity of the key-click signals as the most salient feature. Most information about the key-click

TABLE 7 Information Transfer (in Bits) from Experiment III

\begin{tabular}{c|ccc|c}
\hline Participant & IT_A & IT_F & IT_C & IT(3-D) \\
\hline P1 & 0.56 & 0.40 & 0.13 & 1.69 \\
P2 & 0.75 & 0.66 & 0.28 & 2.09 \\
P3 & 0.73 & 0.71 & 0.13 & 1.85 \\
P4 & 0.76 & 0.42 & 0.01 & 1.59 \\
P5 & 0.57 & 0.03 & 0.01 & 1.11 \\
P6 & 0.53 & 0.29 & 0.06 & 1.26 \\
P7 & 0.83 & 0.75 & 0.02 & 1.88 \\
P8 & 0.47 & 0.14 & 0.02 & 1.07 \\
P9 & 0.82 & 0.57 & 0.07 & 1.83 \\
P10 & 0.64 & 0.69 & 0.10 & 1.78 \\
P11 & 0.33 & 0.11 & 0.03 & 0.84 \\
P12 & 0.55 & 0.08 & 0.03 & 0.99 \\
\hline Average & $\mathbf{0 . 6 3}$ & $\mathbf{0 . 4 0}$ & $\mathbf{0 . 0 7}$ & $\mathbf{1 . 5 0}$ \\
STD & 0.15 & 0.27 & 0.08 & 0.42 \\
\hline
\end{tabular}


signals was carried by signal amplitude, followed by the signal frequency. Very little information was transmitted through the number of cycles. Third, the participants with the higher IT (3D) scores ( $\geq 1.78$ bits; P2, P3, P7, P9, P10) also achieved higher IT_F scores ( $\geq 0.57$ bit) in addition to the higher IT_A cores. It indicates that these participants were able to achieve better identification due to their ability to attend to the frequency as well as the amplitude of the keyclick signals. Finally, the conditional ITs for number of cycles, IT_C, were at 0.13 bit or lower (expect for P2), indicating that little information was available from this parameter for the identification of the key-click signals.

Considerable individual differences can be observed from the IT (3D) values shown in Table 7. While some participants could barely identify 2 signals correctly (e.g., P11 and P12), others are able to correctly identify about 4 signals (e.g., P2, P3, P7). Overall, the results from both the $1 \mathrm{D}$ roving-background and the $3 \mathrm{D}$ identification experiments suggest that some participants could correctly identify 4 out of 12 signals. The confusion matrices also indicated what signals were more salient than others. Feedback from participants implied that more than four signals might be correctly identified if the stimulus alternatives were further reduced by eliminating easily confused signals. For instance, signals A3F1C1 and A2F1P2 were often perceived as the same, and therefore one of them could be eliminated.

Postexperiment participant debriefing led to two additional improvements to the key-click stimulus set with the potential to further improve the overall IT, or equivalently, the total number of perfectly identifiable key-click signals. First, it was noted that some participants had difficulty entering three integers to identify, respectively, the amplitude, frequency, and number of cycles of the key-click signals. Some participants reported that they noticed the crispness of the signal first, followed by the perceived intensity. They then judged the number of cycles by whether there were any perceived "ringing." Therefore, the particular order imposed to our participants for responding to all three parameters was not conducive to high identification accuracy in the 3D identification experiment. Second, as mentioned earlier, it was suggested that some stimulus pairs with high confusion rate should be reduced to one.

To define and evaluate an identifiable simulated key-click signal set, an additional experiment was performed with the three most experienced participants (P1-P3) with 1) a graphic response code that allowed the participants to respond to each stimulus by pressing only one key, and 2) a smaller stimulus set such that for each group of easily confused signals, only one of them was used.

\section{EXPeriment IV: Toward an Identifiable Set of KeY-Click Signals}

The last experiment of the present study was designed with the goal to achieve a near-perfect identification rate so that the stimulus alternatives can be used in a simulated key-click signal set for mobile devices using piezoelectric actuators.

\section{TABLE 8}

Graphic Response Code Used in Experiment IV. The Signal Numbers (S1-S9) Correspond to the Following Parameters: $\mathrm{S} 1=\mathrm{A} 1 \underline{\mathrm{F} 1 \mathrm{C} 2}, \mathrm{~S} 2=\mathrm{A} 1 \mathrm{~F} 1 \mathrm{C} 1, \mathrm{~S} 3=\mathrm{A} 1 \mathrm{~F} 2 \mathrm{C} 1, \mathrm{~S} 4=\mathrm{A} 2 \underline{\mathrm{F} 1 \mathrm{C} 2}$, $\mathrm{S} 6=\mathrm{A} 2 \underline{\mathrm{F} 2 \mathrm{C} 1}, \mathrm{~S} 7=\mathrm{A} 3 \underline{\mathrm{F} 1 \mathrm{C} 2}$, an $\overline{\mathrm{S} 9}=\mathrm{A} 3 \underline{\mathrm{F} 2 \mathrm{C} 1}$

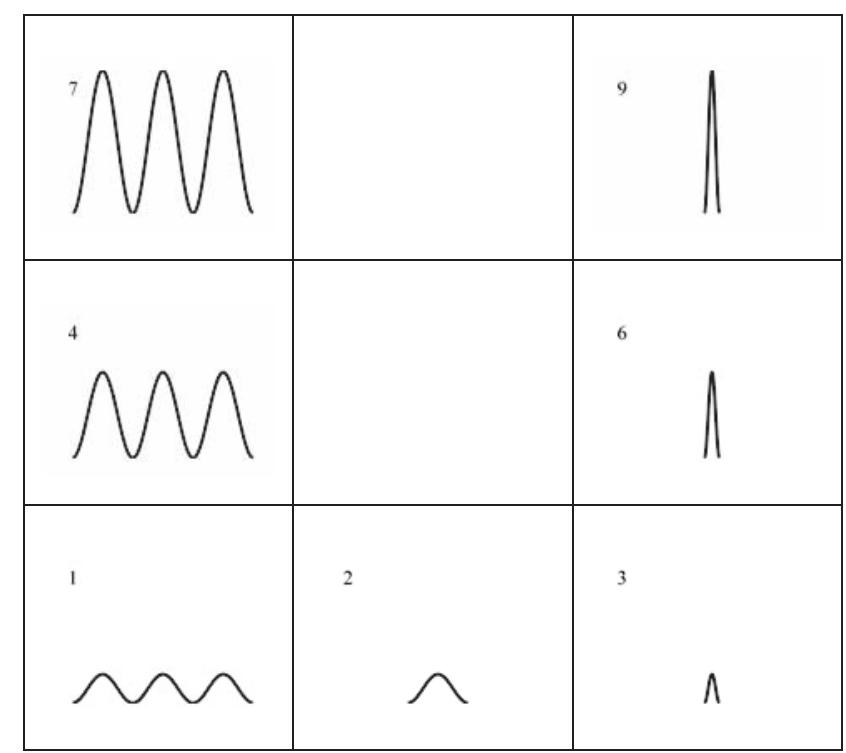

No signal was assigned to the \#5 or \#8 keys.

\subsection{Methods}

From the 12 stimulus alternatives shown in Table 5, we carefully studied the stimulus-response confusion patterns shown in Table 6, and decided to choose the following seven alternatives for our final stimulus set: A1F1C2, A2F1C2, $\mathrm{A} 3 \mathrm{~F} 1 \mathrm{C} 2, \mathrm{~A} 1 \mathrm{~F} 2 \mathrm{C} 1, \mathrm{~A} 2 \mathrm{~F} 2 \mathrm{C} 1, \mathrm{~A} 3 \mathrm{~F} 2 \mathrm{C} 1$, and A1F1C1. With the exception of the last stimulus alternative (A1F1C1), we have correlated C2 (3 cycles) with all the F1 (low frequency) signals (single underline), and C1 (1 cycle) with all the F2 (high frequency) signals (double underline), with the intent to boost the perceived intensity of the low-frequency signals. The signal A1F1C1 turned out to have a distinct feel to it so we decided to keep it in the final stimulus set. Of the five stimulus alternatives used in Experiment III that were eliminated in Experiment IV, A1F2C2, A2F1C1, A2F2C2, and A3F1C1 had the lowest percent-correct scores (85226 correct trials; $11-30$ percent correct; see Table 6). The remaining signal $\mathrm{A} 3 \mathrm{~F} 2 \mathrm{C} 2$ was easily confused with $\mathrm{A} 3 \mathrm{~F} 2 \mathrm{C} 1$, with both having the highest perceived intensities. Although the percent-correct score was higher for A3F2C2 (44 percent) than for $\mathrm{A} 3 \mathrm{~F} 2 \mathrm{C} 1$ (39 percent), we chose to keep the latter to create a better structure for the graphic response code (see below).

The graphic response code consisted of pictures representing the seven stimulus alternatives (see Table 8). They were designed to assist participants in mapping the simulated key-click signals in an intuitive way to ease the process of entering responses. The participants were all recruited from engineering students and staff who were familiar with graphic representations of signals. Therefore, the graphic icons resembled the waveforms sent to the piezoelectric actuator. The three graphic icons in the leftmost column represent the three waveforms at the low frequency (F1) with three amplitudes (A1 to A3). As 
TABLE 9

Pooled Data from Experiment IV

\begin{tabular}{|c|c|c|c|c|c|c|c|c|}
\hline & \multicolumn{7}{|c|}{ RESPONSE } \\
\hline & & $\mathrm{R} 1$ & $\mathrm{R} 2$ & R3 & R4 & R6 & R7 & R9 \\
\hline \multirow{7}{*}{ 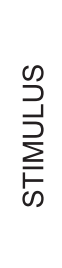 } & S1 & 102 & 3 & 0 & $\underline{6}$ & 0 & 0 & 0 \\
\hline & $\mathrm{S} 2$ & 1 & 107 & 0 & 0 & 0 & 0 & 0 \\
\hline & S3 & 0 & 1 & 115 & 0 & 0 & 0 & 0 \\
\hline & S4 & 12 & 0 & 0 & 90 & 0 & $\underline{3}$ & 0 \\
\hline & S6 & 0 & 0 & 4 & 0 & 98 & 0 & $\underline{2}$ \\
\hline & S7 & 0 & 0 & 0 & $\underline{7}$ & 0 & 93 & 0 \\
\hline & S9 & 0 & 0 & 0 & 0 & $\underline{4}$ & 0 & 102 \\
\hline
\end{tabular}

mentioned earlier, three cycles $(\mathrm{C} 2)$ were associated with all low-frequency stimuli. The three graphic icons in the rightmost column represent the three waveforms at the high frequency (F2) with three amplitudes (A1 to A3) and one cycle (C1). The remaining icon represents A1F1C1. The graphic icons were laid out on a $3 \times 3$ grid and the signal numbers matched the corresponding numbers on the numeric keypad of a keyboard. For example, the participants could press the numeric key " 7 " on the numeric key if the key-click stimulus felt "dull" (F1) and intense (A3).

The experimental procedures were the same as those in Experiment III (3D identification experiment) except for three differences. First, only the three most experienced participants (P1-P3) took part in this follow-up experiment. Second, the graphic code shown in Table 8 was used for entering responses. Third, due to a reduced number of stimulus alternatives, a total of 250 trials, divided into five 50-trial runs, were collected per participant. As before, a training session was available at the beginning of each run. Each participant spent no more than 30 minutes in completing Experiment IV.

\subsection{Results and Discussions}

The stimulus-response confusion matrix pooled from participants P1-P3 is shown in Table 9. The overall percent-correct score was 94 percent. Among the seven stimulus alternatives, signals S2 and S3 were the most distinct with only 1 error trial out of a total 750 trials (99 percent correct). Most confusions occurred between signals S1 and S4, with S1 being misidentified as S4 on 6 trials and S4 as S1 on 12 trials. An additional 10 error trials occurred due to confusion between S4 and S7. The confusion in amplitude level for F1 signals (S1, S4, S7) accounted for 28 (65 percent) of a total of 43 error trials. In comparison, the confusion in amplitude level for F2 signals (S3, S6, S9) totaled 10 trials. The remaining 5 error trials appeared to be random.

The IT(3D) score was 2.5 bits (5.7 items) for each participant. This was a uniform and promising result, indicating that each participant was able to recover 2.5 bits out of a total of 2.8 bits of information available in seven equally likely stimulus alternatives. Equivalently, a maximum set of 5 to 6 simulated key-click signals could be identified without error. Note that these results are better than those shown in the IT (3D) column of Table 7 for the same participants (1.7-2.1 bits), suggesting that IT can be further improved by paring down the total number of stimulus alternatives.
In a subsequent study [1], we studied the efficacy of redundant coding of amplitude and/or frequency information using audio signals presented in synchronization with tactile key-click signals. The results from 12 participants indicated a maximum IT of 2.4 bits, or equivalently, between 5 to 6 perfectly identifiable simulated tactile-audio key-click signals. It thus appears that the human channel capacity for identifying simulated key-click signals using a piezoelectric actuator is limited to 5 to 6 identifiable key clicks.

\section{Concluding Remarks}

We have presented a series of experiments for designing and evaluating a maximum set of identifiable simulated key-click signals for key-less mobile devices. With the continuing reduction in the physical profile of mobile devices, physical keyboards are increasingly being replaced by virtual keys. For many users, however, visual keyboards are less satisfying and sometimes difficult to use than actual pop-dome keys. One solution is to provide simulated key-click signals with a thin layer of piezoelectric actuator to restore the sense of interacting with a tangible keyboard. To the extent that identifiable key-click feedback signals can be achieved with piezos, different key-click sensations can be used to provide contextual cues to users for associating the feel of key clicks with applications such as dialing a phone number versus typing a text message.

Unlike many previous investigations concerning haptic icons and generally the design of haptic signals for mobile devices, the objective of our present study was to design a set of perfectly identifiable key-click signals. Therefore, we pared down stimulus alternatives until identification performance was nearly perfect. Our approach can be summarized as follows: First, we explored the physical parameter space for generating a signal set that span the perceptual space. Second, we conducted 1D and 3D identification experiments (Experiments I-III) to estimate "channel capacity," the largest IT that can be achieved with the signal set. Third, we studied the stimulus-response confusion matrices from the identification experiments to pare down our signal set with the goal to achieve perfect identification performance. Finally, we evaluated the pared-down signal set in another identification experiment (Experiment IV).

We draw the following conclusions and recommendations from the present study. First, of the three physical parameters amplitude, frequency, and number of cycles of raised cosine waveforms, amplitude and number of cycles (when limited to 3 or less) both contribute to the overall perceived intensity of key clicks and therefore cannot be used independently for the purpose of signal identification. Frequency determines the perceived crispness or sharpness of key clicks. In Experiment III, the signals at the higher frequency $(500 \mathrm{~Hz})$ tend to be perceived as stronger than those of the same amplitude at the lower frequency $(125 \mathrm{~Hz})$. If we are not concerned with creating only realistic feeling key clicks, then the number of cycles can increase above three. However, excessive ringings associated with higher number of cycles can sometimes create an eerie impression of something alive underneath the surface of the mobile device.

Second, the results of Experiments I-III indicate that the sum of 1D ITs obtained with roving background (1.3 bits in 
Experiment II) is more predictive of the 3D IT (1.5 bits in Experiment III) than the sum of 1D ITs obtained with fixed background (2.7 bits in Experiment I). Our results therefore support the general additivity law proposed by Durlach et al. [27]. This is a significant finding for future studies as significant reduction in experiment time can be achieved by running several 1D identification experiments instead of one lengthy multi-D identification experiment. Specifically, researchers interested in designing a set of identifiable signals can first conduct a series of 1D identification experiment with roving background, similar to our Experiment II, to assess the overall information transmission capacity. The results can be used to design a small number of perfectly identifiable stimulus alternatives. An evaluation experiment similar to our Experiment IV can then be conducted to verify the new stimulus set. The design process may be iterative and require additional experiments, although it is still relatively fast to conduct $1 \mathrm{D}$ identification experiments and multi-D identification experiments with a small stimulus set.

Third, when the number of alternatives in a signal set was reduced to better match the human channel capacity, an IT of 2.5 bits was achieved in Experiment IV. In terms of the maximum number of key-click signals that can be perfectly identified, it is expected that most people can learn to identify signals S1, S7, S3, and S9 (Table 8) with minimum training, and the more experienced users may be able to handle one or two more signals (i.e., S4 and S6). Therefore, to design haptic key-click simulation on a mobile device using a piezoelectric actuator, one should start with the stimuli shown in Table 8 (cf. Fig. 4 and Table 5 for parameter values) in order to come up with an initial signal set.

Fourth, we observed a nonmonotonic relation between information transfer and task difficulty (number of stimulus alternatives) in the present study. When there were 12 stimuli in Experiment III, the task was still too difficult for the participants, and many mistakes were made due to confusions of perceptually similar stimuli. When the number of stimuli was pared down further in Experiment IV so that it roughly matched the participants' abilities, the task became much easier and performance (in terms of IT) improved. It is, however, not uncommon for human performance to drop precipitously when the difficulty of a task barely exceeds the participant's capability to perform perfectly (e.g., [33], [34]). For example, it was found that when Morse code is presented at a rate that is higher than the receiver's best ability, reception rate (in terms of words per minute) drops precipitously instead of staying at a plateau that corresponds to the receiver's best performance (see Fig. 2 in [33]).

The results of the present study demonstrate our limited ability to process information in an identification task as opposed to a discrimination task. In the end, only 5 to 6 simulated key-click signals can be reliably identified by the most experience participants. Our study also outlines a general methodology for designing and evaluating identifiable signals for other applications. This includes the prediction of multi-D IT from the sum of 1D ITs based on the general additivity law, the use of graphic code to reduce mental load for associating responses to multiattribute stimuli, and a way to reduce stimulus alternatives based on stimulus-response confusion patterns. In general, it appears advisable to use only one or two levels per parameter to generate a identifiable signal set. Our future work will continue to validate the approach used in the present study for creating perceptually identifiable signals for mobile devices and other human-machine interfaces.

\section{ACKNOWLEDGMENTS}

This work was partially supported by a Motorola University Partnership in Research grant.

\section{REFERENCES}

[1] H.-Y. Chen, J. Park, H.Z. Tan, and S. Dai, "Redundant Coding of Simulated Tactile Key Clicks with Audio Signals," Proc. IEEE Haptic Symp., pp. 29-34, 2010.

[2] M. Fukumoto and T. Sugimura, "Active Click: Tactile Feedback for Touch Panels," Proc. Conf. Extended Abstracts on Human Factors in Computing Systems (CHI '01 ), pp. 121-122, 2001.

[3] A. Chang and C. O'Sullivan, "Audio-Haptic Feedback in Mobile Phones," Proc. Conf. Extended Abstracts on Human Factors in Computing Systems (CHI '05), pp. 1264-1267, 2005.

[4] E. Hoggan and S. Brewster, "Designing Audio and Tactile Crossmodal Icons for Mobile Devices," Proc. Ninth Int'l Conf. Multimodal Interfaces, pp. 162-169, 2007.

[5] S. Brewster, F. Chohan, and L. Brown, "Tactile Feedback for Mobile Interactions," Proc. SIGCHI Conf. Human Factors in Computing Systems, pp. 159-162, 2007.

[6] J.C. Lee, P.H. Dietz, D. Leigh, W.S. Yerazunis, and S.E. Hudson, "Haptic Pen: A Tactile Feedback Stylus for Touch Screens," Proc. 17th Ann. ACM Symp. User Interface Software and Technology, pp. 291-294, 2004.

[7] E. Koskinen, T. Kaaresoja, and P. Laitinen, "Feel-Good Touch: Finding the Most Pleasant Tactile Feedback for a Mobile Touch Screen Button," Proc. 10th Int'l Conf. Multimodal Interfaces (ICMI '08), pp. 297-304, 2008.

[8] J. Pasquero, J. Luk, V. Levesque, Q. Wang, V. Hayward, and K.E. MacLean, "Haptically Enabled Handheld Information Display with Distributed Tactile Transducer," IEEE Trans. Multimedia, vol. 9, no. 4, pp. 746-753, June 2007.

[9] P. Laitinen, J. Mawnpaa, and N. Center, "Enabling Mobile Haptic Design: Piezoelectric Actuator Technology Properties in Hand Held Devices," Proc. IEEE Int'l Workshop Haptic Audio Visual Environments and their Applications, pp. 40-43, 2006.

[10] T. Kaaresoja, L.M. Brown, and J. Linjama, "Snap-Crackle-Pop: Tactile Feedback for Mobile Touch Screens," Proc. EuroHaptics '06, 2006.

[11] J. Luk, J. Pasquero, S. Little, K. MacLean, V. Levesque, and V. Hayward, "A Role for Haptics in Mobile Interaction: Initial Design Using a Handheld Tactile Display Prototype," Proc. ACM Conf. Human Factors in Computing Systems (CHI '06), pp. 171-180, 2006.

[12] I. Poupyrev, S. Maruyama, and J. Rekimoto, "Ambient Touch: Designing Tactile Interfaces for Handheld Devices," Proc. 15th Ann. ACM Symp. User Interface Software and Technology (UIST '02), pp. 51-60, 2002.

[13] I. Poupyrev and S. Maruyama, "Tactile Interfaces for Small Touch Screens," Proc. 16th Ann. ACM Symp. User Interface Software and Technology, pp. 217-220, 2003.

[14] V. Hayward and J.M. Cruz-Hernandez, "Tactile Display Device Using Distributed Lateral Skin Stretch," Proc. Haptic Interfaces for Virtual Environment and Teleoperator Systems Symp., pp. 1309-1314, 2000.

[15] L.M. Brown, S.A. Brewster, and H.C. Purchase, "Multidimensional Tactons for Non-Visual Information Presentation in Mobile Devices," Proc. Eighth Conf. Human-Computer Interaction with Mobile Devices and Services, pp. 231-238, 2006.

[16] K.E. MacLean and M. Enriquez, "Perceptual Design of Haptic Icons," Proc. EuroHaptics '03, pp. 351-362, 2003.

[17] B.W. White, F.A. Saunders, L. Scadden, P. Bach-Y-Rita, and C.C. Collins, "Seeing with the Skin," Perception and Psychophysics, vol. 7, pp. 23-27, 1970.

[18] H.Z. Tan and A. Pentland, "Chapter 18: Tactual Displays for Sensory Substitution and Wearable Computers," Fundamentals of Wearable Computers and Augmented Reality, W. Barfield and T. Caudell, eds., pp. 579-598, CRC Press, 2001. 
[19] J.B.F. Erp Van, H.A.H.C. Van Veen, C. Jansen, and T. Dobbins, "Waypoint Navigation with a Vibrotactile Waist Belt," ACM Trans. Applied Perception, vol. 2, pp. 106-117, 2005.

[20] L.A. Jones, B. Lockyer, and E. Piateski, "Tactile Display and Vibrotactile Recognition on the Torso," Advanced Robotics, vol. 20, pp. 1359-1374, 2006.

[21] F.A. Geldard, "Adventures in Tactile Literacy," The Am. Psychologist, vol. 12, pp. 115-124, 1957.

[22] K.E. MacLean, M.J. Enriquez, and T. Lim, "Morphing in Periodic Tactile Signals," World Haptics Conf. '09: Proc. Third Joint EuroHaptics Conf. and Symp. Haptic Interfaces for Virtual Environment and Teleoperator Systems, pp. 178-183, 2009.

[23] W.R. Garner, Uncertainty and Structure as Psychological Concepts. Wiley, 1962.

[24] M. Hollins, R. Faldowski, S. Rao, and F. Young, "Perceptual Dimensions of Tactile Surface Texture: A Multidimensional Scaling analysis," Perception and Psychophysics, vol. 54, pp. 697705, 1993.

[25] M. Zarzo and D.T. Stanton, "Understanding the Underlying Dimensions in Perfumers' Odor Perception Space as a Basis for Developing Meaningful Odor Maps," Attention, Perception, and Psychophysics, vol. 71, pp. 225-247, 2009.

[26] J. Pasquero, J. Luk, S. Little, and K. MacLean, “Perceptual Analysis of Haptic Icons: An Investigation into the Validity of Cluster Sorted MDS," Proc. 14th Symp. Haptic Interfaces for Virtual Environment and Teleoperator Systems, pp. 437-444, 2006.

[27] N.I. Durlach, H.Z. Tan, N.A. Macmillan, W.M. Rabinowitz, and L.D. Braida, "Resolution in One Dimension with Random Variations in Background Dimensions," Perception and Psychophysics, vol. 46, pp. 293-296, 1989.

[28] H.-Y. Chen, "Information Transfer of Tactile Signals on Mobile Devices," Master of Science, Electrical and Computer Eng., Purdue Univ., West Lafayette, Indiana, 2009.

[29] G.A. Miller, "Note on the Bias of Information Estimates," Information Theory in Psychology, H. Quastler, ed., pp. 95-100, The Free Press, 1954.

[30] H.Z. Tan, "Identification of Sphere Size Using the PHANToM ${ }^{\circledR}$ : Towards a Set of Building Blocks for Rendering Haptic Environment," Proc. Sixth Int'l Symp. Haptic Interfaces for Virtual Environment and Teleoperator Systems, vol. 61, pp. 197-203, 1997.

[31] F.G. Ashby and J.T. Townsend, "Varieties of Perceptual Independence," Psychological Rev., vol. 93, pp. 154-179, 1986.

[32] H.Z. Tan, W.M. Rabinowitz, and N.I. Durlach, "Analysis of a Synthetic Tadoma System as a Multidimensional Tactile Display," J. Acoustical Soc. of Am., vol. 86, pp. 981-988, 1989.

[33] H.Z. Tan, C.M. Reed, and N.I. Durlach, “Optimum InformationTransfer Rates for Communication through Haptic and Other Sensory Modalities," IEEE Trans. Haptics, vol. 3, no. 2, pp. 98-108, Apr.-June 2010.

[34] W. Wong and S. Mori, “The Magical 'Wave' Seven, Plus or Minus Two?," J. Acoustical Soc. of Am., vol. 104, pp. 390-398, 1998.

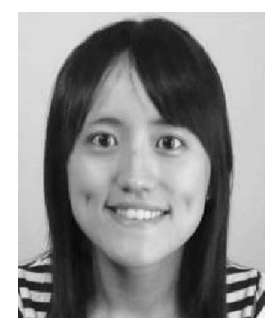

Hsiang-Yu Chen received the BS degree in electrical engineering from National Chiao-Tung University, Hsin-Chu, Taiwan, and the MS degree in electrical and computer engineering from Purdue University, West Lafayette, Indiana. She is currently with Motorola, Inc.

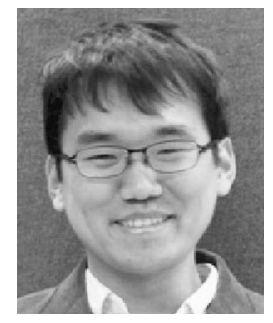

Jaeyoung Park received the BS degree in electrical and computer engineering from Seoul National University, Seoul, Korea, and the MS degree in electrical and computer engineering at Purdue University, West Lafayette, Indiana. He is currently working toward the $\mathrm{PhD}$ degree in electrical and computer engineering at Purdue University.

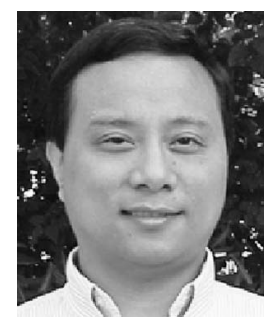

Steve Dai received the bachelor's degree in physics (1985) from East China Normal University and the $\mathrm{PhD}$ degree (1995) in materials science and engineering from the University of Illinois at Urbana-Champaign. He was a distinguished member of Technical Staff at the Motorola Labs and Mobile Devices (19952009). He joined Sandia National Laboratories as a technical staff in 2009. His research interests include novel ceramic materials and applications for wireless communications, micro fluidics, and haptics.

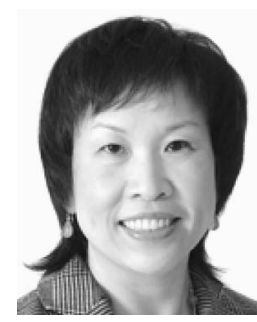

Hong Z. Tan received the bachelor's degree in biomedical engineering (in 1986) from Shanghai Jiao Tong University and received the master's and doctorate degrees (in 1988 and 1996, respectively), both in electrical engineering and computer science, from the Massachusetts Institute of Technology (MIT). She was a research scientist at the MIT Media Lab from 1996 to 1998 before joining the faculty at Purdue University. She is currently an associate professor of electrical and computer engineering, with courtesy appointments in the School of Mechanical Engineering and the Department of Psychological Sciences. She is an associate editor of Presence, ACM Transactions on Applied Perception and IEEE Transactions on Haptics. She served as the founding chair of the IEEE Technical Committee on Haptics from 2006-2008. She was a recipient of the US National Science Foundation (NSF) CAREER award from 2000-2004. Her research focuses on haptic human-machine interfaces in the areas of haptic perception, rendering, and multimodal performance. She is a senior member of the IEEE.

For more information on this or any other computing topic, please visit our Digital Library at www.computer.org/publications/dlib. 